\title{
Slicing and Contours Generation for Fabricating Heterogeneous Objects
}

\author{
Y. K. Siu ${ }^{1}$ and S.T. Tan ${ }^{2}$ \\ Department of Mechanical Engineering \\ The University of Hong Kong,Pokfulam Road,Hong Kong \\ ${ }^{1}$ E-mail :h9610687@hkusua.hku.hk \\ ${ }^{2}$ E-mail :sttan@hkucc.hku.hk
}

\begin{abstract}
A contour sub-division algorithm on each layer arising from slicing a heterogeneous object is proposed. Within each slice, the material grading is decomposed into subcontours according to the different grading variation. A parameter called "grading step-width" is defined to control the number of sub-contours and resolution of the grading. With such discretization, it is, therefore, possible to build a heterogeneous object on layered manufacturing machines of different fabricating precision specification.
\end{abstract}

\section{Introduction}

Layered manufacturing (LM) technologies for fabricating a part with continuously changing material composition throughout the part reduces the gap between what a designer wants and what can be made with current manufacturing technologies. With this type of LM techniques, it would be possible to design and make a part optimally using the different desired properties of the materials involved to suit the application requirements, be it for structural, thermal or aesthetic reasons.

Several technologies have been developed for fabricating heterogeneous objects. Fessler et al. [1] improved some commonly used layered manufacturing (LM) machines such as the shape deposition manufacturing (SDM) laser deposition system in order to fabricate functionally graded metals through the use of powder mixing. Jackson et al. [2] also developed a 3D printer for fabricating heterogeneous objects. It is similar to the ink-jet printer, using different nozzle (printer head) to print different materials/binders by a "drop-by-drop" manner in order to fabricate a heterogeneous object.

Concurrently, several modeling schemes for heterogeneous objects have also been proposed $[3,4]$ and they allow designers to design not only the geometry, but also the material distribution of an object. The CAD model is thus enriched with useful material information. However, there lacks a simple data (both material and geometry) format link between the CAD system and the LM machines. In order to provide a more general format for the LM machines, a simple post-processing on the heterogeneous solid model may be necessary. One of the possible solutions is to truncate a continuous material grading into a simple step-wise grading. The techniques describe in this paper may form the basis for an information exchange between CAD models and the LM machines.

\section{Previous Work - Heterogeneous solid modeling system}

To model a heterogeneous object with material variations, a CAD system should first be able to know the material composition of each point of the object. A material array $M$ is defined for mapping the material composition information to every point of the object. It is defined that each element of the material composition array $M$ represents the volume fraction of a pre-defined primary material and the total volume fraction of the primary materials for the material composition arrays should be summed to one.

$\sum_{j=1}^{n} M_{j}=1$

where,

$M_{j}=j$-th element of the material composition array $M$

$n \quad$ number of primary materials

For example, if $M=(0 \%, 30 \%, 20 \%, 50 \%)$ stands for a given point $\underline{x}$, the material composition of $\underline{x}$ is $0 \%$ material A, $30 \%$ material $\mathrm{B}, 20 \%$ material $\mathrm{C}$ and $50 \%$ material D.

In a material grading, the material composition of an object varies from one end to another which are stored in the material composition arrays $\mathrm{M}_{\mathrm{s}}$ and $\mathrm{M}_{\mathrm{e}}$. By relating the two end material composition arrays and the material grading function $f(d)$, the material composition grading can be represented by the following equation. 


$$
\begin{array}{ll}
v_{j}=M_{s j} & \text { for } f(d)<0 \\
v_{j}=f(d) \times\left(M_{e j}-M_{s j}\right)+M_{s j} & \text { for } 0 \leq f(d) \leq 1 \\
v_{j}=M_{e j} & \text { for } f(d)>1
\end{array}
$$

where,

$M_{s j}=\mathrm{j}$-th element of the material composition array $M_{s}$ $M_{e j}=\mathrm{j}$-th element of the material composition array $M_{e}$ $v_{j}=$ the volume fraction for the $\mathrm{j}$-th primary material during grading

The material grading function $f(d)$ is a distance function of material grading of a geometric point from a reference datum (also termed as "grading source"). The reference could be a fixed reference in the coordinate system of the model, such as a point, a line, a plane or the outer most boundaries of a model. Figure 1 shows the effects of grading and the definition of $d$ in the grading function $f(d)$ in the different types of grading sources.

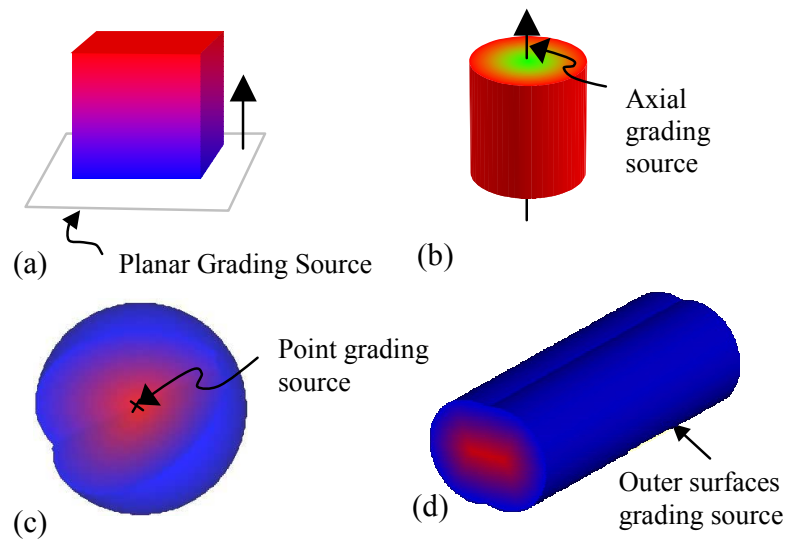

Figure 1. (a) $d=$ distance of a point from a reference plane

(b) $d=$ radial distance of a point from a reference axis

(c) $d=$ distance of a point from a reference point

(d) $d=$ minimum distance of a point from the selected outer surfaces

According to the material grading function $f(d)$, three regions in the heterogeneous object are defined for each grading source $G$ inside a heterogeneous object. A point $\underline{x}$ in the Euclidean space $\mathrm{A}^{3}$ can fall in one and only one of the following regions.
$G_{-c}^{3}=\left\{\underline{x} \in A^{3} \mid f(d)<0\right\}$

iii.) +ve Complementary Region,

$G_{+c}^{3}=\left\{\underline{x} \in A^{3} \mid f(d)>1\right\}$

The function of the two complementary regions is to make sure that the material compositions of every point inside the object are defined. The material composition of each point in the heterogeneous object can be described by a material composition array and $B$ is used to define the collection of those material arrays within a specific region according to equation (2).

$B_{\text {eff }}=f(d) \times\left(M_{e}-M_{s}\right)+M_{s}$

$B_{-c}=M_{s}$

$B_{+c}=M_{e}$

where,

$B_{\text {eff }}$ denotes the material distribution in the Effective Grading Region,

$B_{-c}$ denotes the material distribution in the $-v e$ Complementary Region, and

$B_{+c}$ denotes the material distribution in the +ve

Complementary Region,

Therefore, for each grading source $G$, we can pair up the geometric grading regions and the material composition arrays of these regions respectively.

$G=\left(\left(G_{e f f}^{3}, B_{e f f}\right),\left(G_{-c}^{3}, B_{-c}\right),\left(G_{+c}^{3}, B_{+c}\right)\right)$

An example is shown in Figure 2. A detailed description of the modeling scheme is presented in [4]

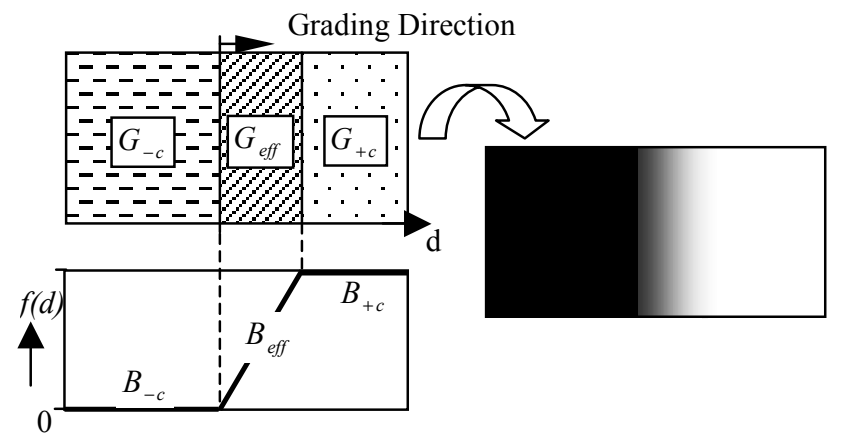

Figure 2. Model of a heterogeneous object with a planar grading source
i.) Effective Grading Region, $G_{e f f}^{3}=\left\{\underline{x} \in A^{3} \mid 0 \leq f(d) \leq 1\right\}$
ii.)-ve Complementary Region, 


\section{Contour sub-division on material grading}

Jackson et al. [2] developed a 3D printer for fabricating heterogeneous objects. The working principle of this 3D printer is similar to the common ink-jet printers, i.e. the printer head prints different materials in each slice in a drop-by-drop manner. Similar to ink-jet printing, 3-D printing is a binary device. During the printing process, it can only decide either "to print" or "not to print", i.e. a "black and white" bitmap. The continuous material grading is converted into a discrete version of machine instructions by a technique of digital half-toning as shown in Figure 3.
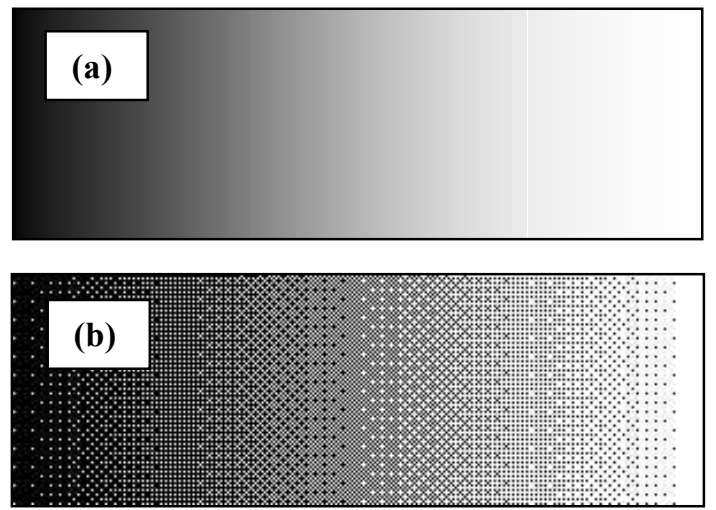

Figure 3. (a) Original material grading

(b) Half-toned grading

The digital half-tone printing suffers from the disadvantages of requiring an enormous amount of storage space and precision problem because it is difficult to fabricate heterogeneous objects with material distributions varying from point to point like printing a bitmap. Moreover, the resultant material properties may not be the expected one because the materials are not well mixed internally before printing.

Apart from the "drop-by-drop" method of Jackson et al., another widely used method includes a variety of powder layering techniques [5]. Under these techniques, the continuous material grading is discretized into stepwise gradings. The materials are mixed into a desired volume ratio and built step-by-step according to the different material compositions of the grading. Compared with the grading done in the ink-jet "drop-by-drop" manner, discretized grading is simpler, easier and more economical to implement. Besides, as the materials are well mixed before fabrication, it can help to avoid undesired excursions from the as-planned composition profile.
In addition to reasons mentioned above, the digital half-toning method might not be optimal for the powder layering techniques since it may not be practical and realistic for composite manufacturing like FGM. A more general layered representation is thus necessary. Apart from storing each layer as an array of pixels or voxels as in [2], some other common sliced formats can also be used. These commonly used file formats consist of successive cross-sections taken at ascending $\mathrm{Z}$ intervals in which solid material is represented by interior and exterior boundary polylines (contours). CLI (Common Layer Interface) and SLC (Stereolithography Contour) are two of the commonly used file formats.

Although these file formats are unable to represent material information inside the contours, they can be modified and an algorithm for generating contours which supports material information can be established. The basic idea of the algorithm is to sub-divide each slice of a heterogeneous object into a list of multi-material contours according to the grading functions $f(d)$ of different grading sources.

\subsection{Contours generation}

As mentioned in section 2, three regions compose a grading source. Since the material compositions inside the $+\mathrm{ve}$ and $-\mathrm{ve}$ complementary regions are constant throughout, contour sub-division is only necessary in the effective grading region. To sub-divide a continuous material grading, a "grading step-width" parameter $t_{g}$ is defined $\left(0<t_{g} \leq 1\right)$ to control the resolution of the grading. In this way, a heterogeneous object can be built by layered manufacturing machines of different fabricating precision specification. In other words, depending on the precision of the fabricating machines, each slice of the heterogeneous object can be converted to a required resolution using the $t_{g}$ parameter.

The number of contours that can be generated within the effective grading regions depends on $t_{g}$. The smaller the step-width, the larger is the number of the contours generated. The number of contours in the effective grading region is given by, $\mathrm{N}_{\mathrm{c}}=\frac{1}{t_{\mathrm{g}}}+1$.

The grading function $f(d)$ intensity of the different contours during sub-division are defined by the following equations: 


$$
\begin{array}{ll}
f(d)=0 & \text { For } 0 \leq f(d)<\frac{t_{g}}{2} \\
f(d)=t_{g} \times(\mathrm{u}+1) & \text { for }\left(\frac{1}{2}+\mathrm{u}\right) \times t_{g} \leq f(d)<\left(\frac{1}{2}+\mathrm{u}+1\right) \times t_{g} \\
& \text { and } \mathrm{u}=0,1,2, \ldots \mathrm{N}_{\mathrm{c}}-3, \mathrm{u} \geq 0 \\
f(d)=1 & \text { for }\left(\frac{1}{2}+\mathrm{N}_{\mathrm{c}}-2\right) \times t_{g} \leq f(d) \leq 1
\end{array}
$$

The material composition of each step is redefined according to the truncated $f(d)$ equation. The equations ensure that when the step-width is set to maximum (i.e. $t_{g}$ $=1$ ), the grading can be eventually truncated into a multiple material object where material boundary is shown in Figure 4(d).

For example, if $t_{\mathrm{g}}=0.25, \mathrm{~N}_{\mathrm{c}}=\frac{1}{0.25}+1=5$ contours are formed.

$$
\begin{array}{ll}
f(d) & =0 \\
f(d) & =t_{g} \times 1 \\
f(d) & =t_{g} \times 2 \\
f(d) & =t_{g} \times 3 \\
f(d) & =1
\end{array}
$$$$
\begin{aligned}
& \text { for } 0 \leq f(d)<t_{g} \times 0.5 \\
& \text { for } t_{g} \times 0.5 \leq f(d)<t_{g} \times 1.5 \\
& \text { for } t_{g} \times 1.5 \leq f(d)<t_{g} \times 2.5 \\
& \text { for } t_{g} \times 2.5 \leq f(d)<t_{g} \times 3.5 \\
& \text { for } t_{g} \times 3.5 \leq f(d) \leq 1
\end{aligned}
$$

The sub-division of the grading is shown in Figure 4(c).

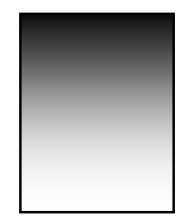

(a) Original grading

$$
\left(t_{g} \rightarrow 0\right)
$$

Figure 4. Sliced contour with different step-widths

From the above example, it is seen that by varying the step-width, the material grading can be altered from a simple multiple material case with sharp material boundary $\left(t_{g}=1\right)$ to one with smooth grading $\left(t_{g} \rightarrow 0\right)$.

A model shown in Figure 5(a) is used to demonstrate the contour sub-division algorithm of a slice. First, each slice is formed by sampling the material composition of a lattice of points within the geometric boundaries of each layer of the heterogeneous object. The $f(d)$ intensity map is thus generated as shown in Figure 5(b).
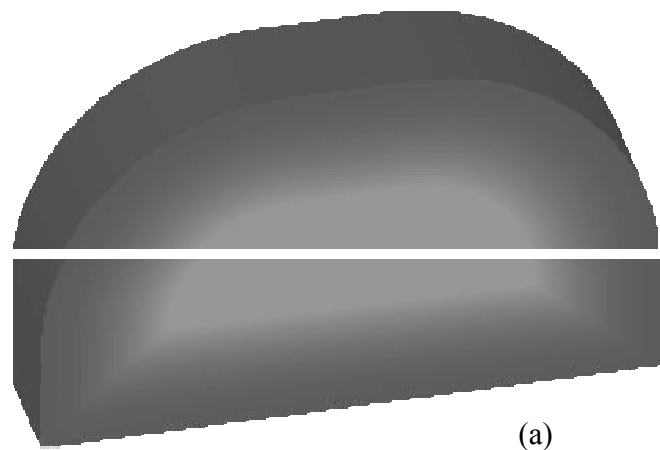

(a)

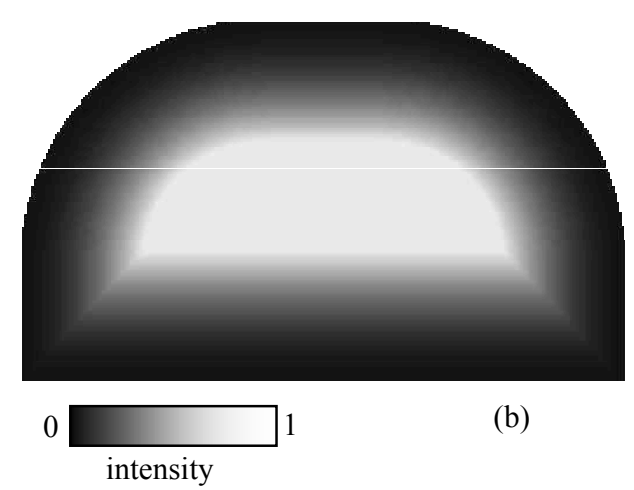

Figure 5.

(a) a heterogeneous model

(b) an intensity map $f(d)$ of the slice in Figure $5(a)$

Figure 6 shows that the continuous material grading in the effective grading region is truncated into a stepwise grading by specifying the step-width $t_{g}=0.25$.

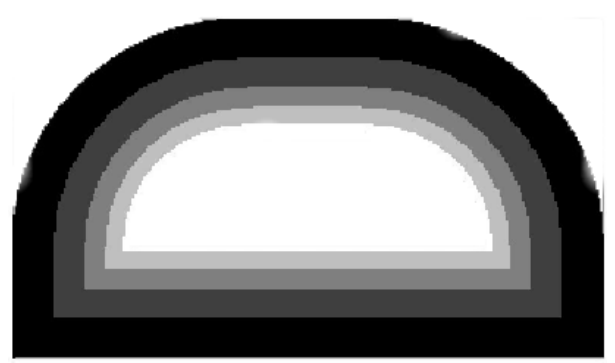

Figure 6. Truncated stepwise grading

\subsubsection{Homogeneous region}

For each contour $\mathrm{C}$, a material composition array $\mathrm{M}$ is associated to it. The material composition within contour $\mathrm{C}$ remains constant through the region inside $\mathrm{C}$, we so call it a "homogeneous region". The homogeneous region is not necessary to contain a single material only, the term "homogeneous" refers to the average material composition within the region as shown in Figure 7. 


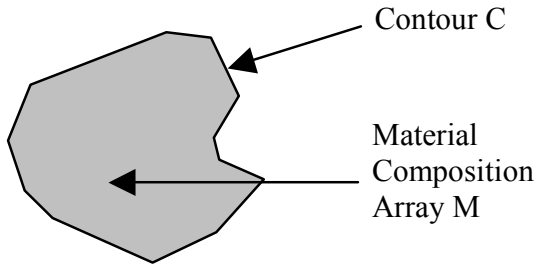

Figure 7 Homogeneous region

Contours of the three grading regions are then generated accordingly. The total number of generated contours is the summation of the contours generated in these three grading regions. A series of contours are then formed as shown in Figure 8.

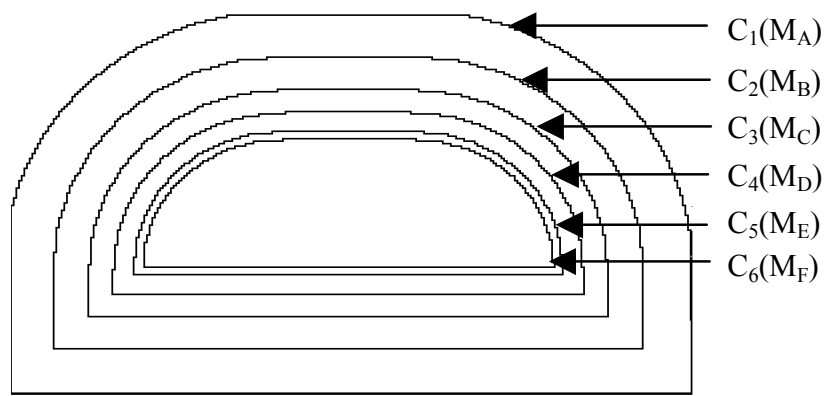

Figure 8. Total number of contours generated according to the truncated $f(d)$

As each contour $\mathrm{C}$ has its own material composition array $\mathrm{M}$, the problem becomes a multiple material object's slice representation which has been elaborated in [6]. Based on the previous study, a tree structure (Figure 9) is generated to extract the material composition of the contours in each slice. $\mathrm{C}_{\mathrm{i}}\left(\mathrm{M}_{\mathrm{A}}\right)$ means that the material composition of contour $\mathrm{C}_{\mathrm{i}}$ is stored in a material array $\mathrm{M}_{\mathrm{A}}$. This example generates a simple tree structure, while in the case of multiple grading sources, a more complicated tree will be created.<smiles>CC(C)(C)[Mg]</smiles>

Figure 9. Tree structure of contours

\subsubsection{Fabrication Example}

From the contours generated in Figure 8 and the contour tree in Figure 9 for determining the nesting property, the continuous material grading profile of a heterogeneous model is converted into slices of discretized gradings as shown in Figure 10.

Figure 11 shows the effect of using different grading step-width $t_{\mathrm{g}}$. The prototypes are fabricated using a Color $3 \mathrm{D}$ printer by Z-Corp.

\subsection{Contours extraction for multiple grading sources}

The effect of combining different grading sources of different material grading in a heterogeneous object via an intersecting operator " $\otimes$ ". The intersecting operator " $\otimes$ " is defined to sum up the material composition of the intersecting regions. A detailed description of the intersecting operator is presented in [4]

$M_{1} \otimes_{k 1 k 2} M_{2}=\frac{k_{1} M_{1}+k_{2} M_{2}}{k_{1}+k_{2}}$

where

$\mathrm{k}_{\mathrm{i}}$ is the weighting ratio of the grading sources

$\mathrm{M}_{\mathrm{i}}$ is the material composition of the homogeneous regions

Upon slicing the object and on each layer, the grading sources are decomposed into step-wise grading. Different step-widths $\left(t_{\mathrm{g}}\right)$ can be given to different grading sources and contours for the effective grading regions of different grading sources are generated independently as described in section 3.1. Each contour $\mathrm{C}$ in a slice contains a homogeneous material region $R$ within which the material composition is the same.

$R=(\mathrm{C}(\mathrm{M}), \mathrm{I})$

where,

$\mathrm{C} \in$ points on the contour of the region with material composition array $\mathrm{M}$,

$\mathrm{I} \in$ points inside the region

Intersection between grading sources leads to intersection of contours in a layer during the slicing process. In order to ensure that there is no overlapping between contours, those overlapping regions, which are caused by intersection of grading sources, are extracted out. A new contour and the material composition of this region is generated. For example, if $\mathrm{C}_{I}\left(\mathrm{M}_{\mathrm{A}}\right)$ and $\mathrm{C}_{2}\left(\mathrm{M}_{\mathrm{B}}\right)$ are contours belonging to grading source $G_{1}$ and $G_{2}$ respectively, each contour has its own material 
composition stored in a two-material composition arrays $\mathrm{M}_{\mathrm{A}}$ and $\mathrm{M}_{\mathrm{B}}$.

$$
R_{1}=\left(\mathrm{C}_{1}\left(\mathrm{M}_{\mathrm{A}}\right), \mathrm{I}_{1}\right), R_{2}=\left(\mathrm{C}_{2}\left(\mathrm{M}_{\mathrm{B}}\right), \mathrm{I}_{2}\right)
$$

On intersection, three new regions are defined. The material compositions and contours of these regions are regenerated.

Intersecting region $R_{12}=R_{1} \cap^{*} R_{2}=\left(\mathrm{C}_{12}{ }^{\prime}\left(\mathrm{M}_{\mathrm{A}} \otimes \mathrm{M}_{\mathrm{B}}\right), \mathrm{I}_{12}{ }^{\prime}\right)$ $R_{1}{ }^{\prime}=R_{1} l^{*} R_{12}=\left(\mathrm{C}_{1}{ }^{\prime}\left(\mathrm{M}_{\mathrm{A}}\right), \mathrm{I}_{1}{ }^{\prime}\right)$

$R_{2}{ }^{\prime}=R_{2} /^{*} R_{12}=\left(\mathrm{C}_{2}{ }^{\prime}\left(\mathrm{M}_{\mathrm{B}}\right), \mathrm{I}_{2}^{\prime}\right)$

$\left(/^{*}\right.$ and $\cap^{*}$ denote the regularized difference and intersection operators)

A schematic diagram of extracting the intersecting contour is shown in Figure 12.

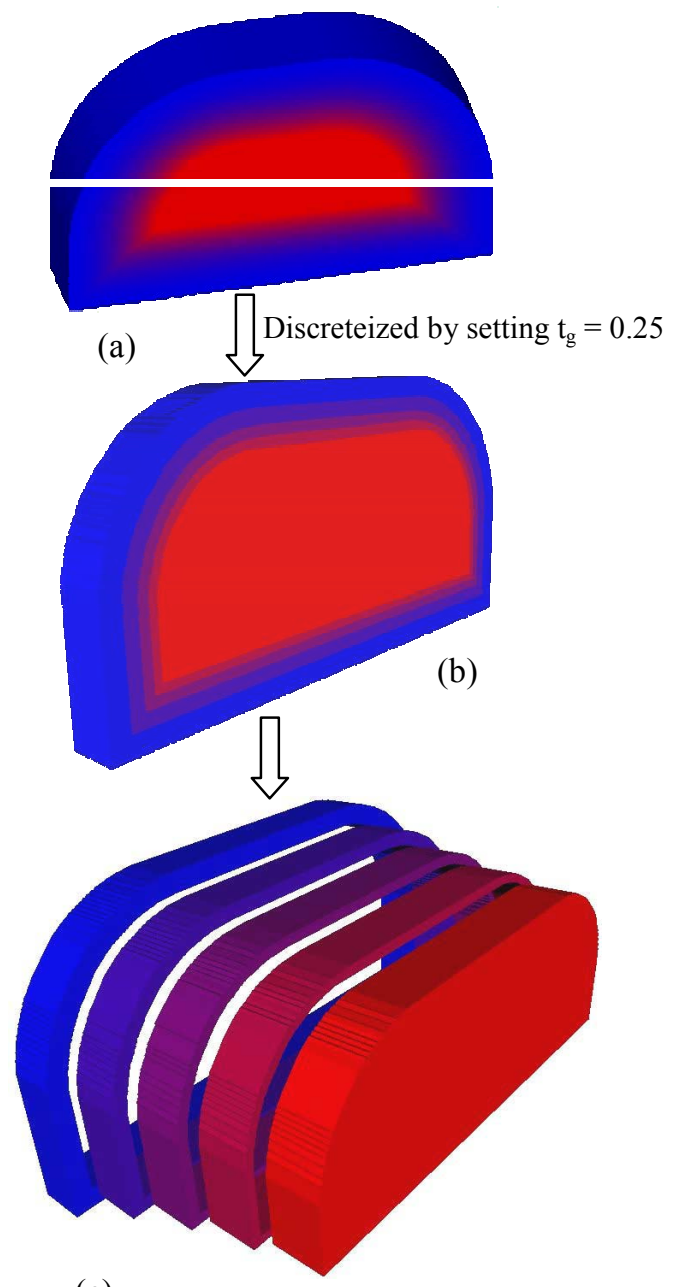

(c)

Figure 10. (a) a heterogeneous object model

(b) discreteizd version of the model

(c) decomposed into multiple materials object

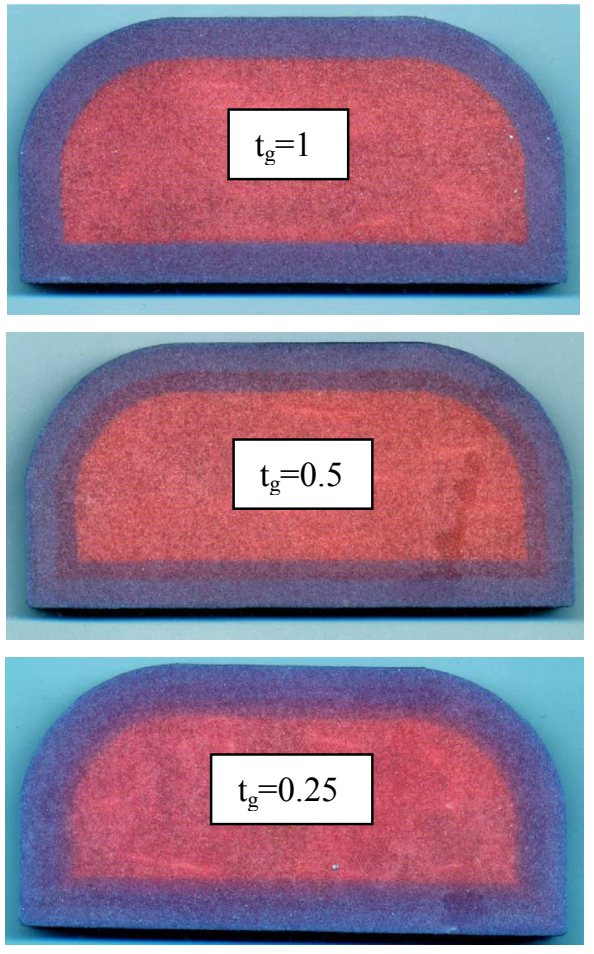

Figure 11 Fabricated examples using different grading step width

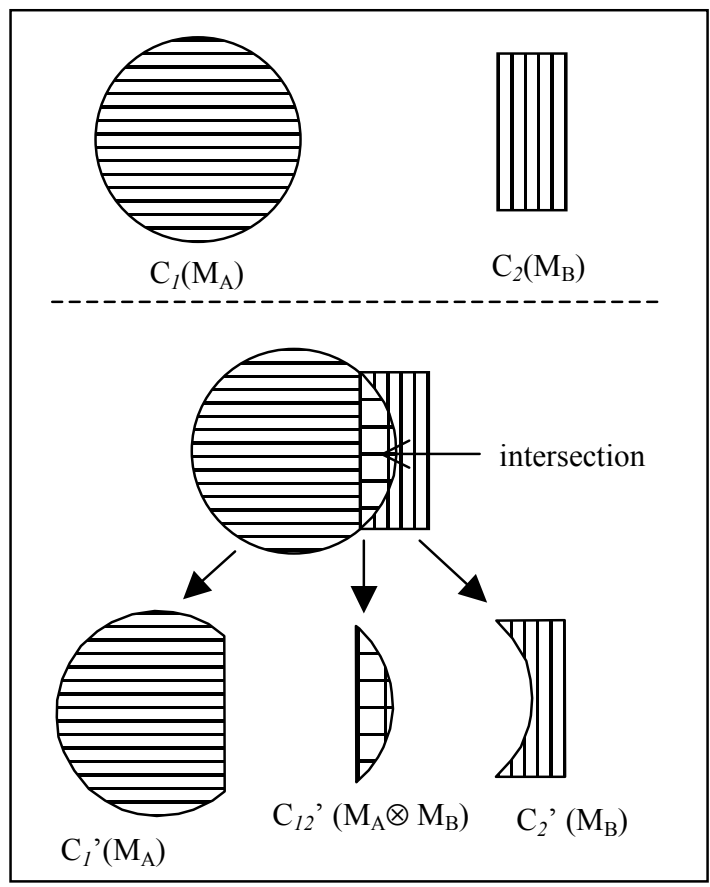

Figure 12. Schematic diagram of extracting the intersecting contour 
Three new sub-contours, $\mathrm{C}_{12}{ }^{\prime}\left(\mathrm{M}_{\mathrm{A}} \otimes \mathrm{M}_{\mathrm{B}}\right), \mathrm{C}_{1}{ }^{\prime}\left(\mathrm{M}_{\mathrm{A}}\right)$ and $\mathrm{C}_{2}{ }^{\prime}\left(\mathrm{M}_{\mathrm{B}}\right)$ are formed and a new tree structure is regenerated according to the new contours list.

\section{Conclusion}

In this paper, a contour sub-division method by truncating the material grading from continuous grading to step-wise grading for fabrication is discussed. By adjusting the "grading step-width" $t_{g}$, the precision for fabrication can be adjusted. In addition, a sample using different colors to simulate different materials have been built in order to verify scheme. The representation scheme and examples of using the proposed modeling scheme are implemented on the Unigraphics V16 CAD system.

\section{Acknowledgment}

The authors would like to thank the Department of Mechanical Engineering, The University of Hong Kong and the Research Grant Council for supporting this project.

\section{References}

[1] J. Fessler, A. Nickel, G. Link, F. Prinz, P. Fussell, "Functional Gradient Metallic Prototypes through Shape Deposition Manufacturing", Proceedings of the Solid Freeform Fabrication Symposium, August 1997.

[2] T. R. Jackson, H. Liu, N. M. Patrikalakis, E. M. Sachs, and M. J. Cima, "Modeling and Designing Functionally Graded Material Components for Fabrication with Local Composition Control", Materials and Design, Vol. 20, No. 2/3, pp.63-75, June 1999

[3] V. Kumar and D. Dutta, "An Approach to Modeling Heterogeneous Objects", ASME Journal of Mechanical Design, Vol. 120, No. 4, December 1998

[4] Y. K. Siu and S.T. Tan, "Source-based" heterogeneous solid modeling", CAD Journal, Vol. 34, pp. 41-55, 2002

[5] J. M. Whitney and R. L. Mccullough, "Delaware Composites Design Encyclopedia - Volume 2/Micromechanical materials modeling", Technomic Publishing Company, 1989

[6] W. K. Chiu and S. T. Tan, "Multiple Material Objects: From CAD Representation to Data Format for Rapid Prototyping", CAD, Vol. 32, No. 12, pp. 707-718, 2000 